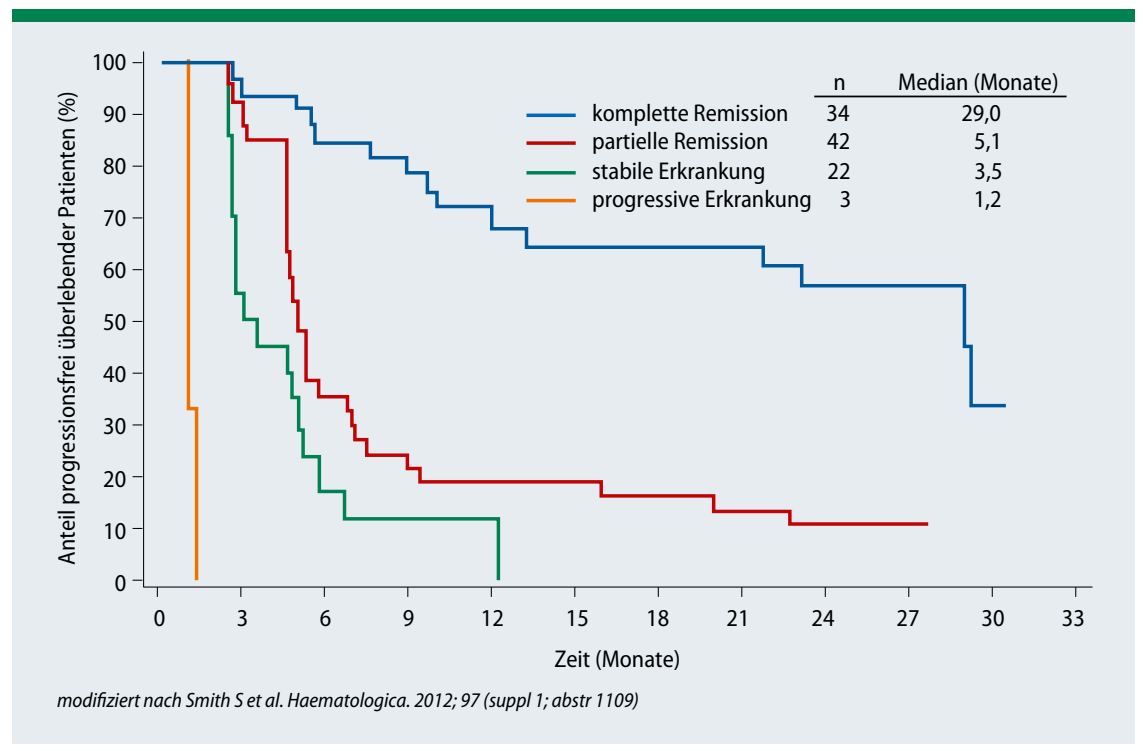

Abb. 1: Progressionsfreies Überleben unter Brentuximab Vedotin in Abhängigkeit vom klinischen Ansprechen

ca. 2012; 97 (suppl 1; abstr 0542)]. Eine zweite Kohorte aus 61 Patienten mit rezidivierter oder refraktärer CLL oder SLL (Small Lymphocytic Lymphoma) hatte zuvor bereits mindestens zwei Therapien erhalten, eine davon mit Fludarabin, und 44 von ihnen $(72 \%)$ wiesen mindestens ein Hochrisiko-Merkmal auf: Deletion 17 p oder $11 \mathrm{q}$ bzw. unmutierter IGHVStatus. Diese Patienten bekamen entweder $420 \mathrm{mg} / \mathrm{d}(\mathrm{n}=27)$ oder die doppelte Dosis $(840 \mathrm{mg} / \mathrm{d})$.

Ibrutinib wurde gut vertragen, und in der Kohorte mit rezidivierter/refraktärer CLL war die Wirksamkeit mit einer Ansprechrate von etwa zwei Dritteln erstaun- lich hoch, ein weiteres knappes Viertel der Patienten zeigte ein überwiegend nodales Ansprechen. Das ist offenbar typisch für Ibrutinib: In den ersten beiden Monaten der Behandlung scheint sich die Tumorlast überwiegend vom Lymphknoten- ins periphere Kompartiment zu verschieben. Patienten mit genetischen Risikofaktoren zeigen kein deutlich schlechteres Ansprechen.

Die progressionsfreie Überlebensrate nach zwölf Monaten lag in der 420-mgGruppe bei $88 \%$, bei den therapienaiven Patienten, die die gleiche Dosierung bekommen hatten, waren es nach 15 Monaten $96 \%$, und auch die Ansprechrate war hier mit $81 \%$ (davon $12 \%$ komplette Remissionen) für eine Monotherapie beträchtlich; dazu kamen noch einmal 12\% der Patienten mit nodalem Ansprechen. Sollten sich diese Ergebnisse in weiteren Studien bestätigen, sei möglicherweise mit einem Paradigmenwechsel in der Therapie der CLL zu rechnen, prognostizierte O’Brien.

\section{CML: Tiefe Remission korreliert mit besserem klinischem Outcome}

Bei der chronischen myeloischen Leukämie (CML) sind Tyrosinkinase-Inhibitoren (TKI) inzwischen so wirksam, dass schon Studien gestartet wurden, in denen untersucht wird, ob eine Remission auch nach Absetzen der Medikamente erhalten bleibt - was möglicherweise einer Heilung entsprechen würde. Diese Versuche basieren auf Studien wie der ENESTndStudie, in der der Zweitgenerations-TKI Nilotinib gegenüber dem alten Standard Imatinib getestet wurde und $\mathrm{zu}$ der in Amsterdam die 3-Jahres-Resultate vorgestellt wurden. Von den neu diagnostizierten Patienten mit CML in der chronischen Phase erreichten nach drei Jahren $73 \%$ eine gute molekulare Remission (MMR), d. h. ein Absinken der BCR-ABLTiter nach dem Internationalen Standard auf höchstens $0,1 \%$; $32 \%$ hätten sogar eine komplette molekulare Remission, eine $\mathrm{MR}^{4,5}$ (BCR-ABL $\leq 0,0032 \%$ ), erzielt, erklärte Richard Clark, Liverpool, Großbritannien. Im Imatinib-Arm waren es nur 53 bzw. $15 \%$ [Clark R et al. Haematologica. 2012; 97 (suppl 1; abstr 0583)].

\title{
Nachgefragt bei Prof. Dr. med. Stephan Stilgenbauer
}

? Wie schätzen Sie die Entwicklung der Therapie bei der CLL derzeit ein?

Stilgenbauer: Was wir in letzter Zeit und insbesondere auch bei diesem EHA-Kongress gesehen haben, sind extrem vielversprechende Daten für Substanzen, die in den Signalweg des B-Zell-Rezeptors eingreifen: Hier hat man bei sehr stark vortherapierten Patienten, die refraktär gegen Chemotherapie sind, trotzdem ein erstaunlich gutes Ansprechen und dabei nur extrem geringe Nebenwirkungen gesehen. Am weitesten fortgeschritten ist hier die klinische Entwicklung des BTKInhibitors Ibrutinib, aber Ähnliches gilt auch für Inhibitoren der Phosphoinositol-3-Kinase (PI3K) sowie für einige Apoptose-modulierende Substanzen.
? Würden Sie der US-Amerikanerin Susan O'Brien aus Houston zustimmen, dass wir möglicherweise vor einem Paradigmenwechsel in der CLL-Therapie stehen?

Stilgenbauer: Hier kann man Susan O'Brien nur zustimmen. Obwohl die Daten noch sehr neu sind, kann man festhalten, dass über die letzten Jahre und Jahrzehnte noch keine neuartige Therapieoption mit derartig guten Wirksamkeits- und Verträglichkeitsdaten aufwarten konnte. Wenn sich diese Ergebnisse bestätigen, wird ein Paradigmenwechsel - weg von der guten alten Chemotherapie und hin zu gezielten, an der Biologie der Erkrankung ansetzenden Behandlungen - in naher Zukunft vollziehbar sein.

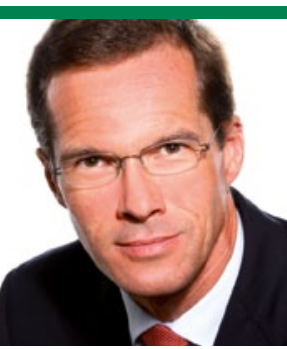

Prof. Dr. Stephan Stilgenbauer

Klinik für Innere Medizin III, Zentrum für Innere Medizin, Universitätsklinikum Ulm 